\title{
Measurement of the Structure of the Proton at HERA
}

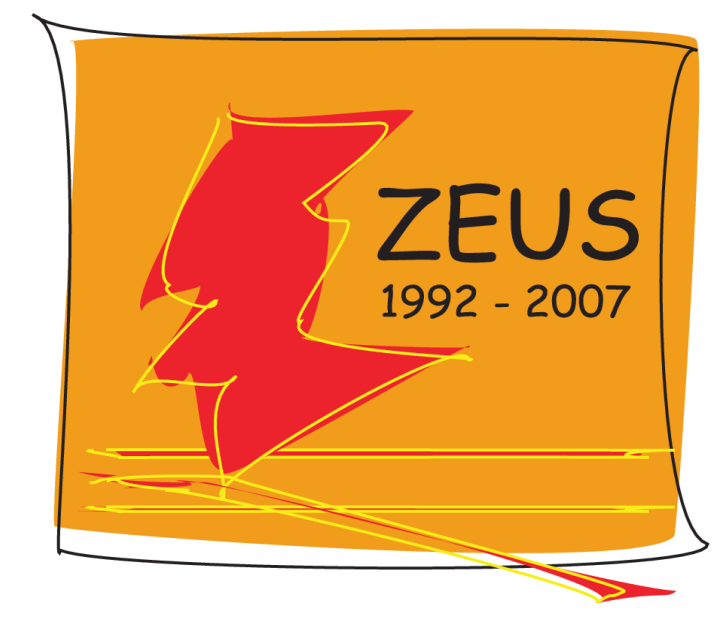

Michał Własenko University of Bonn

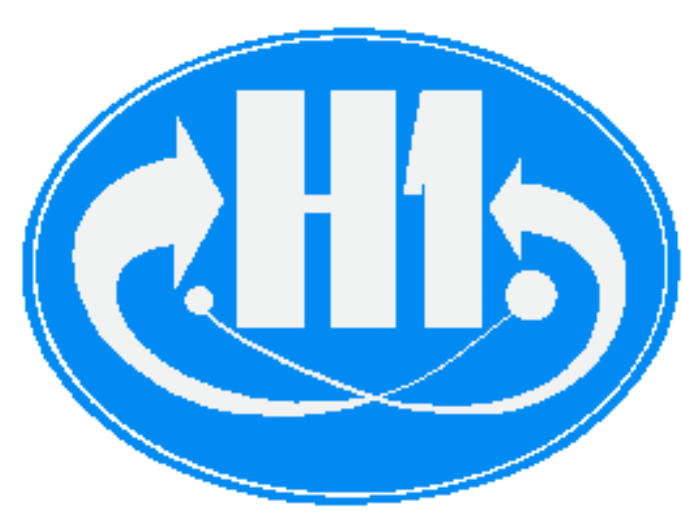

\section{OUTLINE:}

- NC and CC cross sections

- HERA combined PDF fits

- First direct measurements of $F_{L}$ 


\section{The HERA collider}

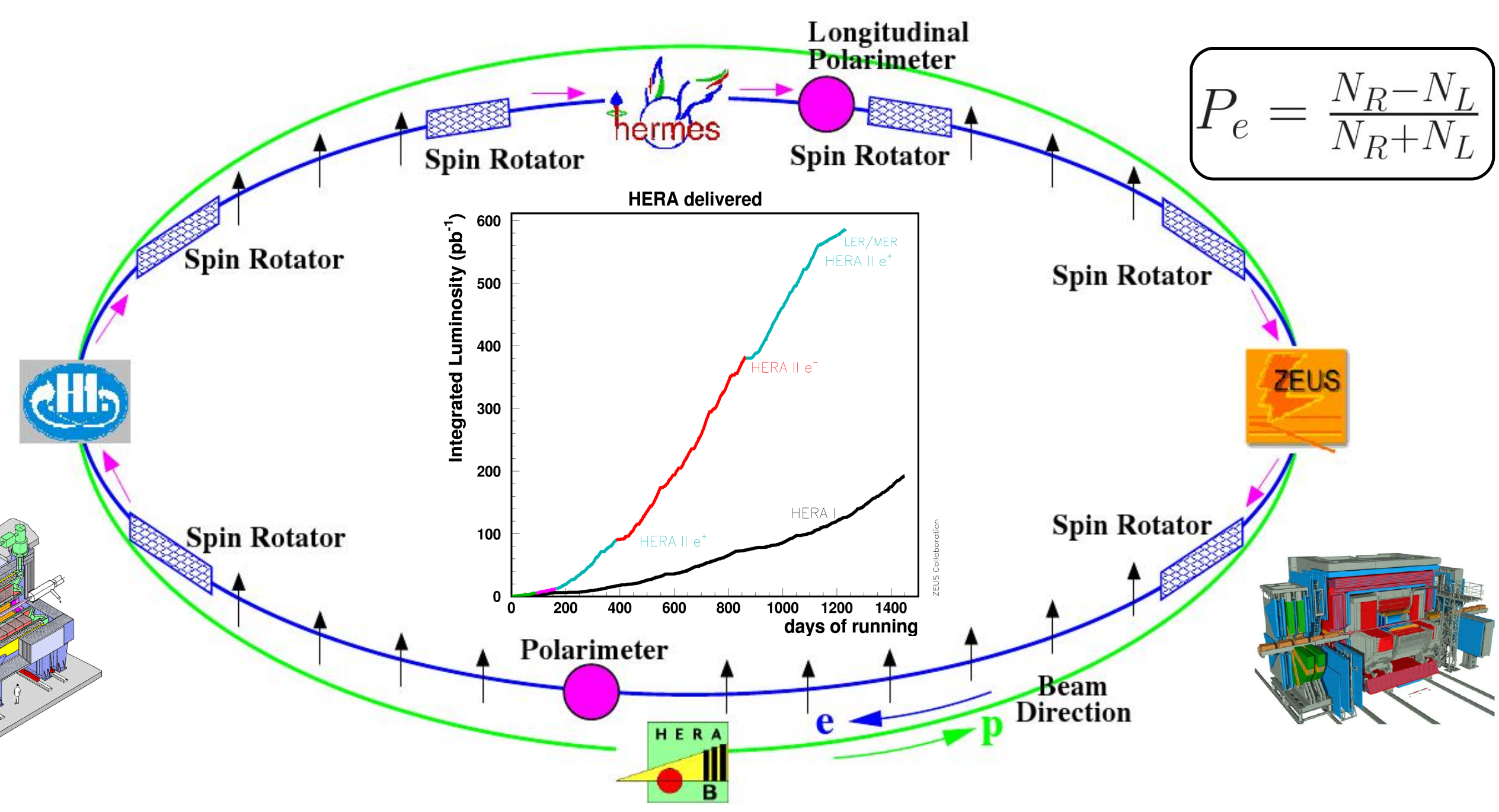

- $E_{p}=920 \mathrm{GeV}, E_{\mathrm{e}}=27.5 \mathrm{GeV}, \sqrt{ } \mathbf{s} \approx 320 \mathrm{GeV}$ (high-energy runs)

- Polarized lepton beams at HERA2, average $\boldsymbol{P}=\mathbf{3 0 - 4 0 \%}$

- Total recorded luminosity: $\mathbf{0 . 5} \mathbf{f b}^{-1}$ by both collaborations

Proton Structure from HERA, Michał Własenko 


\section{NC Deep Inelastic Scattering}
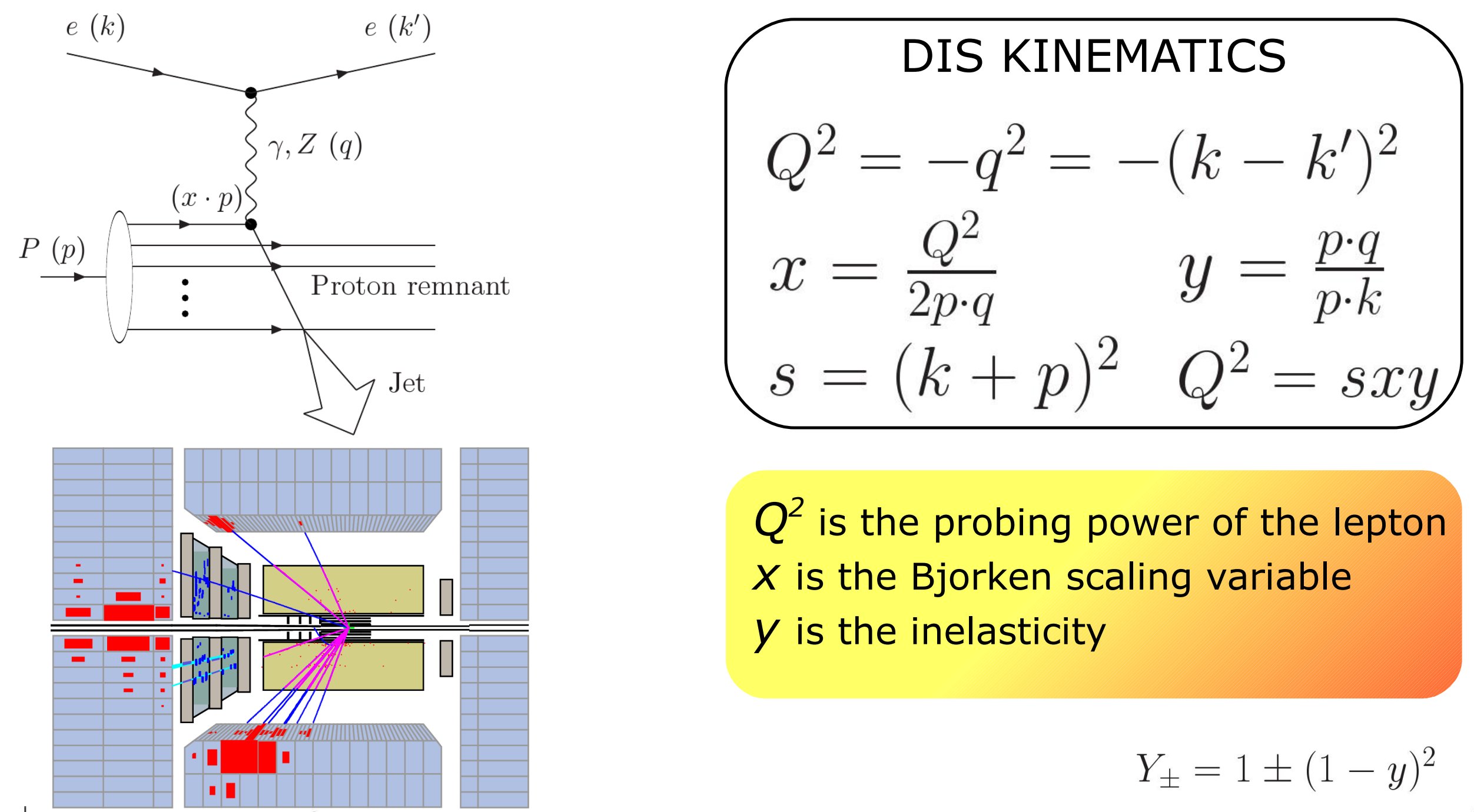

$Q^{2}$ is the probing power of the lepton $X$ is the Bjorken scaling variable $y$ is the inelasticity

$\frac{d^{2} \sigma_{N C}^{ \pm}}{d x d Q^{2}}=\frac{2 \pi \alpha^{2}}{x Q^{4}}\left(Y_{+} \tilde{F}_{2}\left(x, Q^{2}\right) \mp Y_{-} x \tilde{F}_{3}\left(x, Q^{2}\right)-y^{2} \tilde{F}_{L}\left(x, Q^{2}\right)\right)$ 


\section{NC Structure Functions}

$$
\begin{aligned}
& \begin{array}{c}
\text { dominant } \\
\text { contribution }
\end{array} \begin{array}{c}
\mathbf{e}^{ \pm} \\
\text {polarization }
\end{array} \\
& \tilde{F}_{2}=F_{2}^{\gamma}-\left(v_{e}-P_{e} a_{e}\right) \chi_{Z} F_{2}^{\gamma Z}+\left(v_{e}^{2}+a_{e}^{2}-2 P_{e} v_{e} a_{e}\right) \chi_{Z}^{2} F_{2}^{Z} \\
& x \tilde{F}_{3}=-\underbrace{\left(a_{e}-P_{e} v_{e}\right)}_{\text {axial-vector \& vector }} \chi_{Z} x F_{3}^{\gamma Z}+\underbrace{\left(2 v_{e} a_{e}-P_{e}\left(v_{e}^{2}+a_{e}^{2}\right)\right) \chi_{Z}^{2} x F_{3}^{Z}}_{\text {sensitivity only at high } \mathbf{Q}^{2}} \\
& \chi_{Z}=\frac{1}{\sin ^{2} 2 \theta_{W}} \frac{Q^{2}}{M_{Z}^{2}+Q^{2}} \\
& \text { couplings of } \mathrm{e}^{ \pm} \text {to } Z_{0}
\end{aligned}
$$

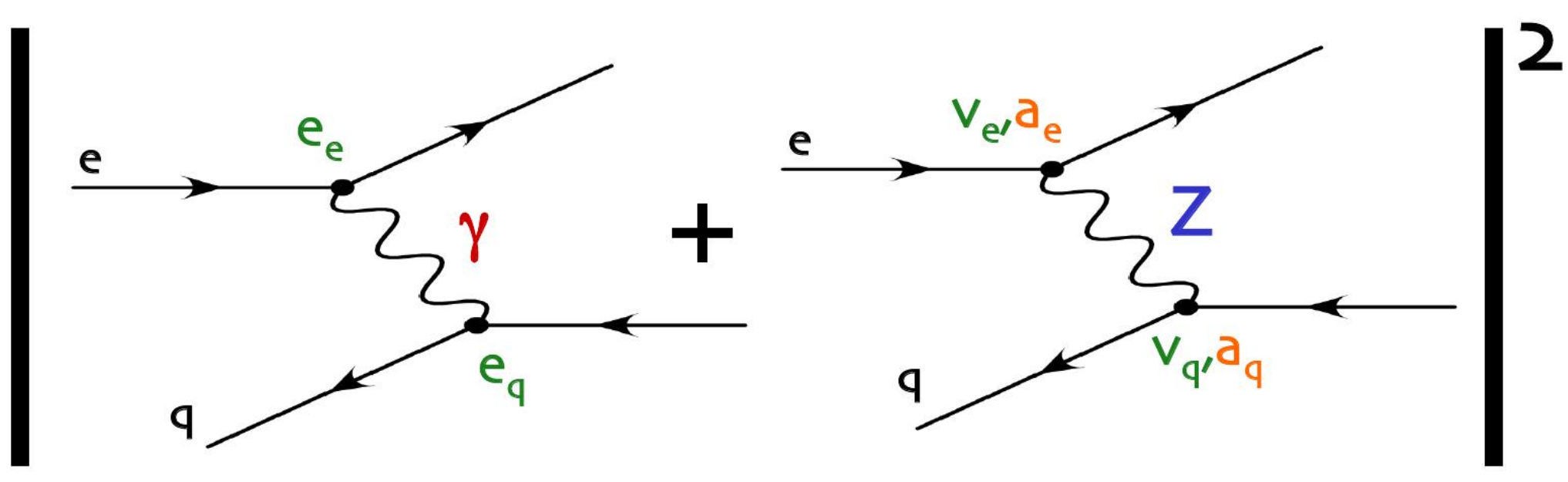

Proton Structure from HERA, Michał Własenko 


\section{NC Structure Functions}

$$
\begin{aligned}
& \begin{array}{c}
\text { dominant } \\
\text { contribution }
\end{array} \begin{array}{c}
\mathbf{e}^{ \pm} \\
\text {polarization }
\end{array} \\
& \tilde{F}_{2}=F_{2}^{\gamma}-\left(v_{e}-P_{e} a_{e}\right) \chi_{Z} F_{2}^{\gamma Z}+\left(v_{e}^{2}+a_{e}^{2}-2 P_{e} v_{e} a_{e}\right) \chi_{Z}^{2} F_{2}^{Z} \\
& x \tilde{F}_{3}=-\underbrace{\left.-a_{e}-P_{e} v_{e}\right)}_{\text {axial-vector \& vector }} \times \underbrace{}_{Z F_{3}^{\gamma Z}}+\underbrace{\left(2 v_{e} a_{e}-P_{e}\left(v_{e}^{2}+a_{e}^{2}\right)\right) \chi_{Z}^{2} x F_{3}^{Z}}_{\text {sensitivity only at high } \mathbf{Q}^{2}} \\
& \text { couplings of } e^{ \pm} \text {to } z_{o} \\
& \chi_{Z}=\frac{1}{\sin ^{2} 2 \theta_{W}} \frac{Q^{2}}{M_{Z}^{2}+Q^{2}}
\end{aligned}
$$

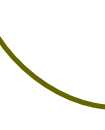

$$
\begin{aligned}
& {\left[F_{2}^{\gamma}, F_{2}^{\gamma Z}, F_{2}^{Z}\right]=\sum_{q}\left[e_{q}^{2}, 2 e_{q} v_{q}, v_{q}^{2}+a_{q}^{2}\right] x(q+\bar{q}) \begin{array}{c}
\text { sea \& val } \\
\text { quarks }
\end{array}} \\
& {\left[x F_{3}^{\gamma Z}, x F_{3}^{Z}\right]=\sum_{q}\left[e_{q} a_{q}, v_{q} a_{q}\right] 2 x(q-\bar{q}) \text { valence quarks }} \\
& x g(x)=1.8\left(\frac{3 \pi}{2 \alpha_{s}} F_{L}(0.4 x)-F_{2}(0.8 x) \backsim \frac{8.3}{\alpha_{s}} F_{L} \begin{array}{c}
\text { gluons via } \boldsymbol{F}_{L} \\
\text { at high y }
\end{array}\right.
\end{aligned}
$$

Proton Structure from HERA, Michał Własenko 


\section{HERA combined NC cross sections $\left(F_{2}\right)$}

$$
\sigma_{r}^{e^{ \pm} p \rightarrow e^{ \pm} X}=F_{2}-\frac{y^{2}}{Y_{+}} F_{L} \mp \frac{Y_{-}}{Y_{+}} x F_{3}
$$

HERA I $e^{+} p$ Neutral Current Scattering - H1 and ZEUS

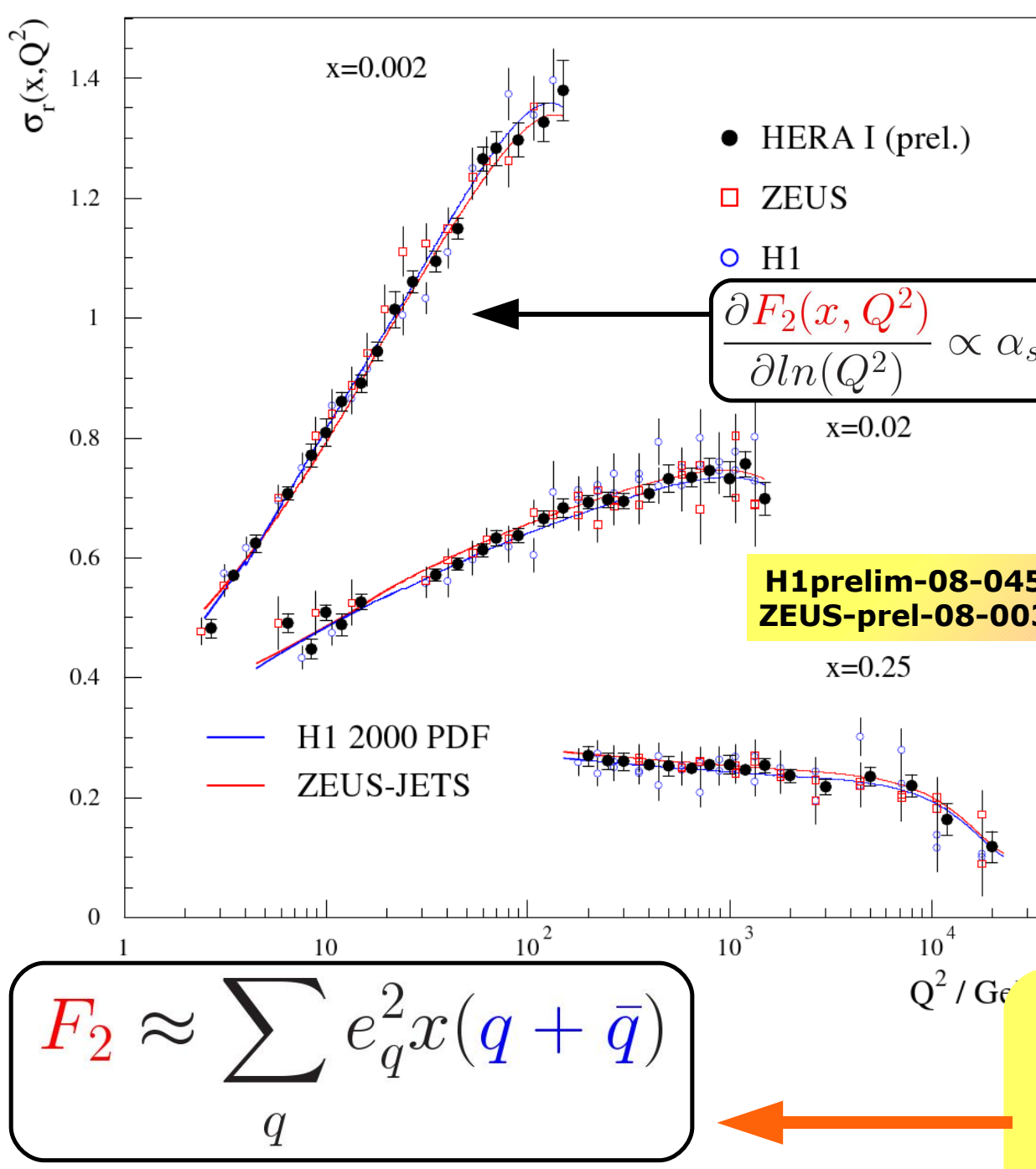

Proton Structure from HERA, Michał Własenko
H1 and ZEUS Combined PDF Fit

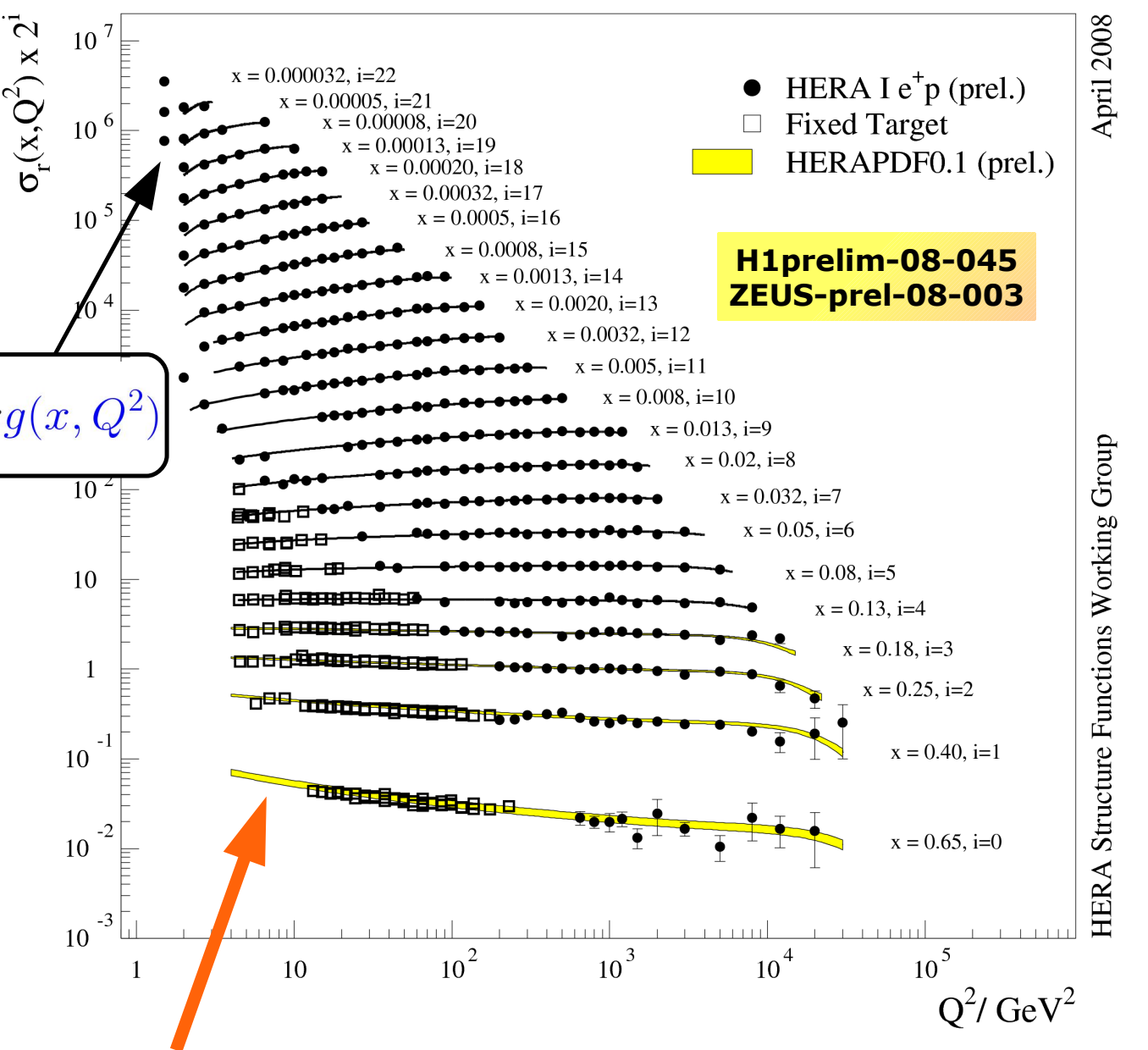

- HERAI precision: 2-3\% (syst. limited)

- NLO QCD precisely describes $F_{2}$ over 4 orders of magnitude in $\left(x, Q^{2}\right)$ including scaling violation 


\section{PDF fits on HERAI data}
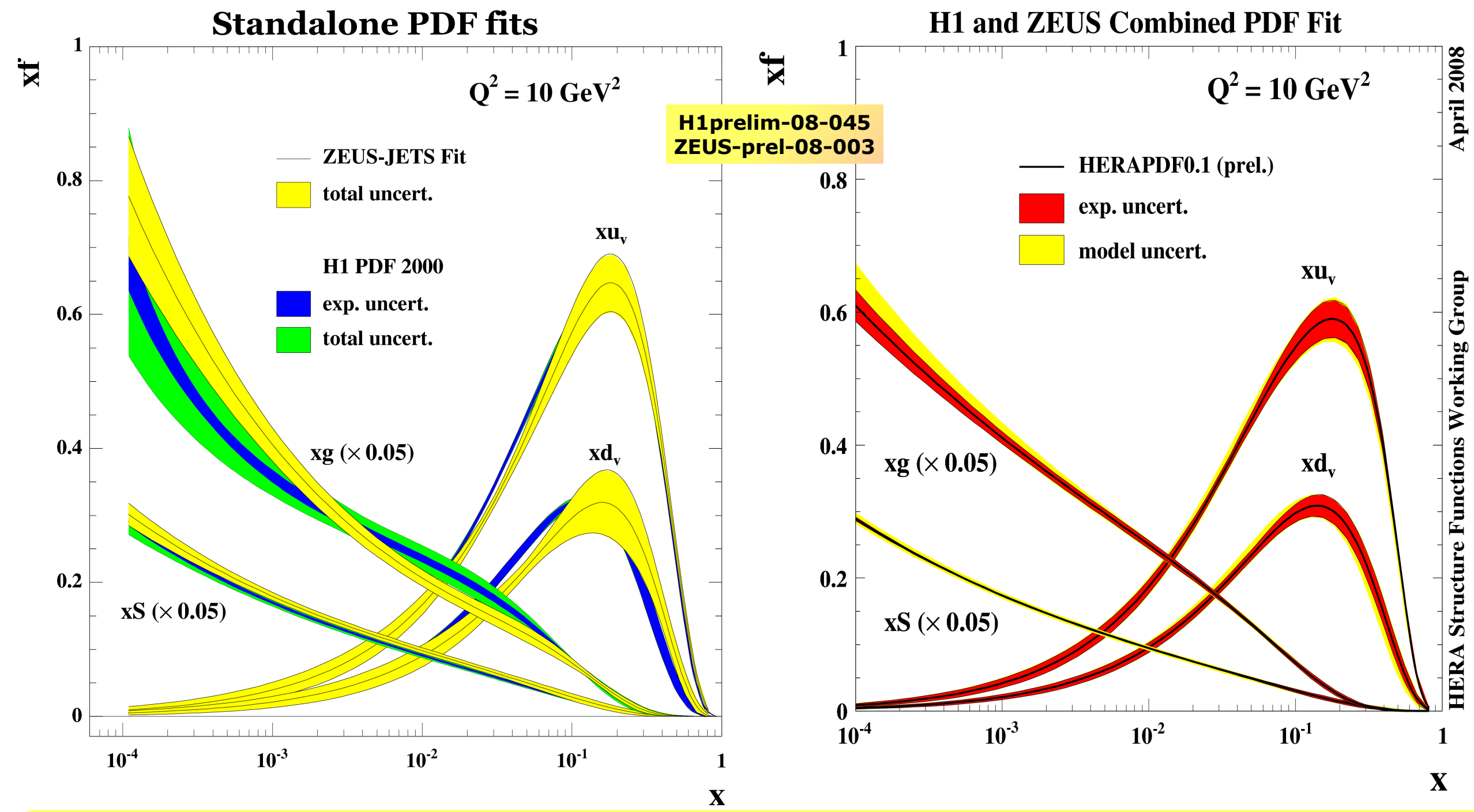

- Standalone fits compatible over the whole HERA kinematic plane, with $\boldsymbol{g}(\boldsymbol{x})$ a bit different

- After $\sigma\left(x, Q^{2}\right)$ averaging (model-independent way), $\chi^{2} / \mathbf{N D F}=\mathbf{4 7 7 / 5 6 2}$ for the PDF fit 


\section{Comparison to Global Fits}
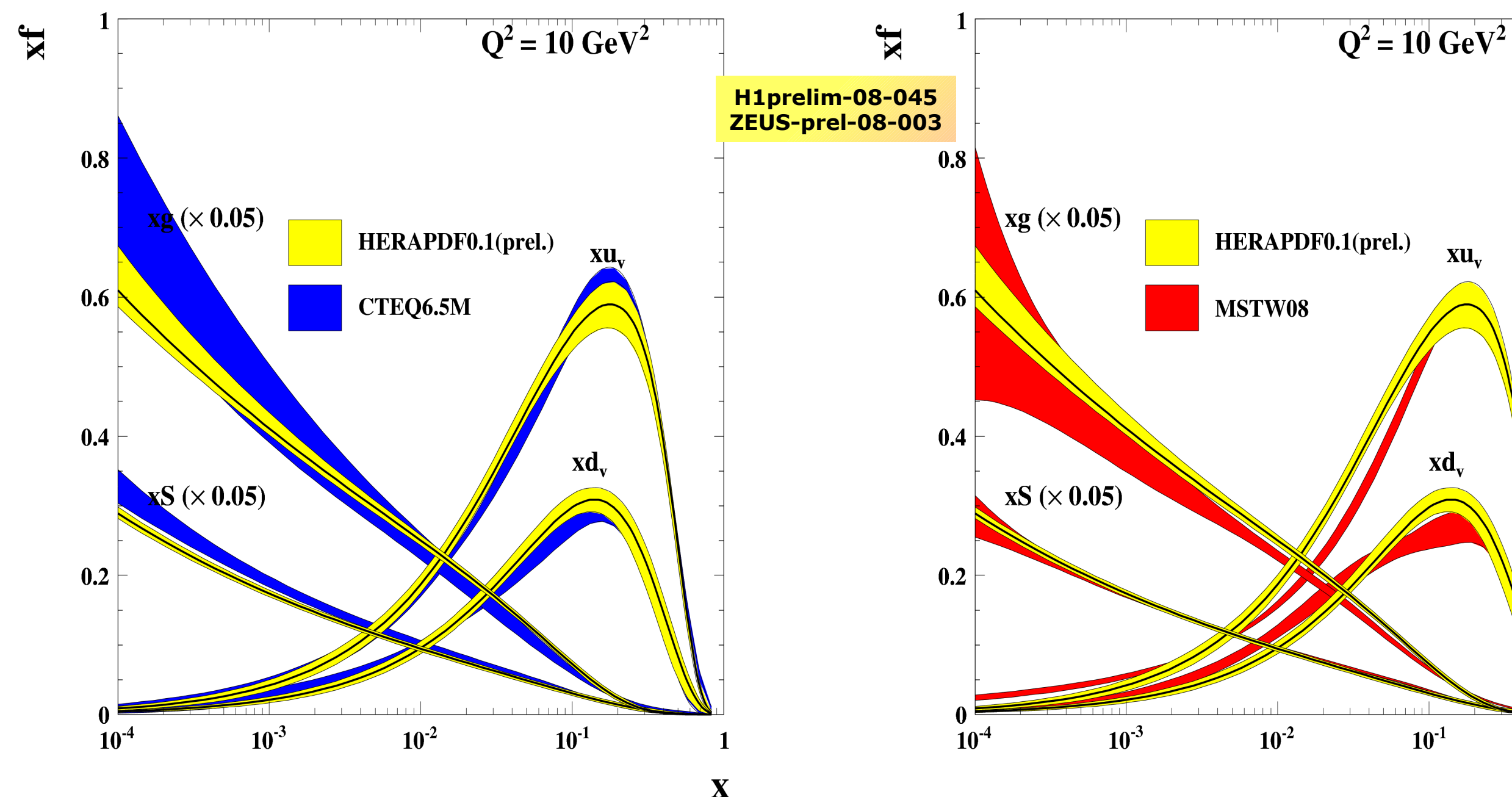

H1prelim-08-045 ZEUS-prel-08-003

- Compared to the recent Global fits, HERA PDFs offer tighter uncertainties

- Global fits, however involve a much larger variety of input experiments

- HERAPDF fits library available (via LHAPDF), providing valuable input for Tevatron and LHC

Proton Structure from HERA, Michał Własenko

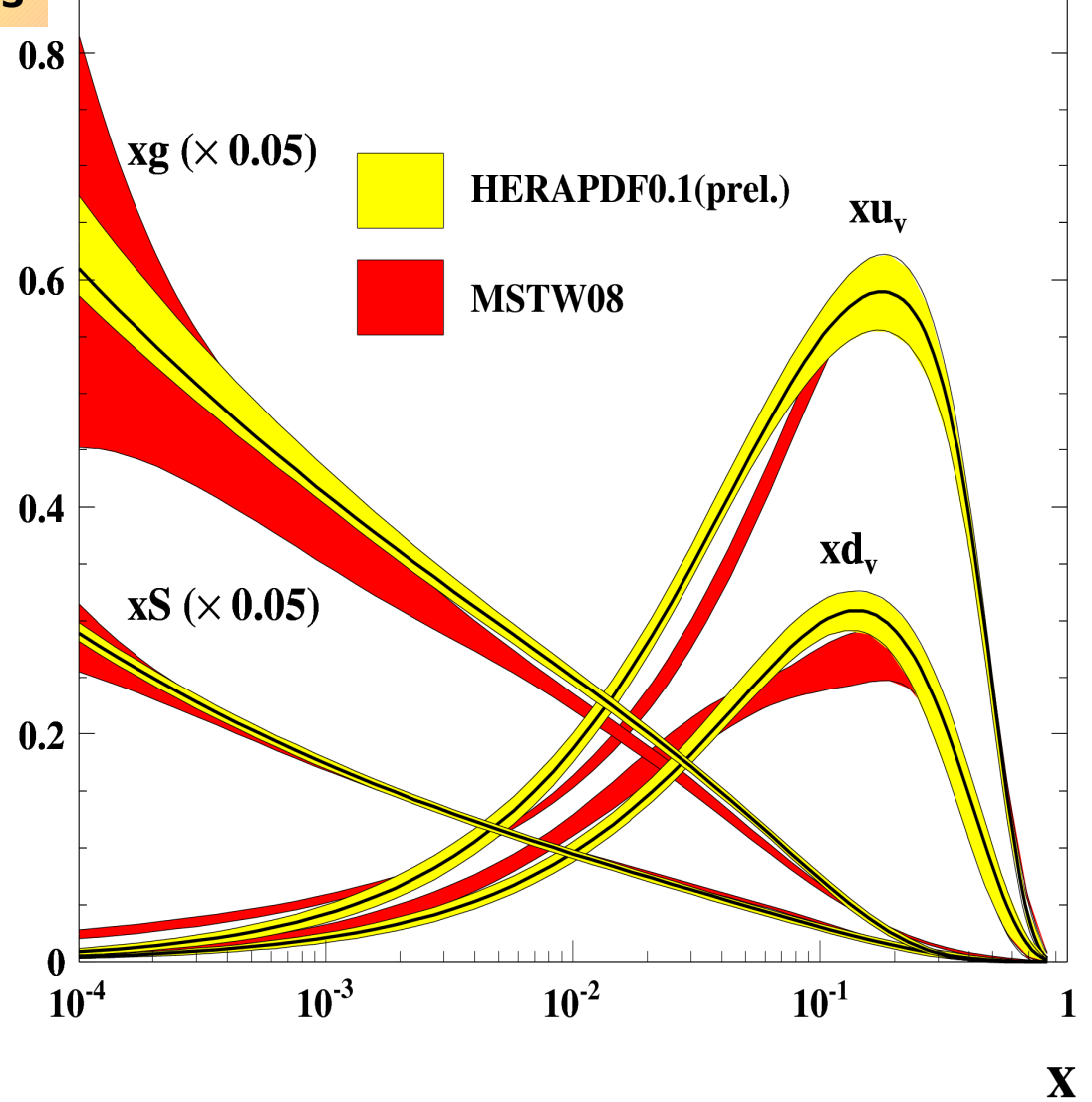




\section{Unpolarized high $Q^{2}$ NC cross sections}

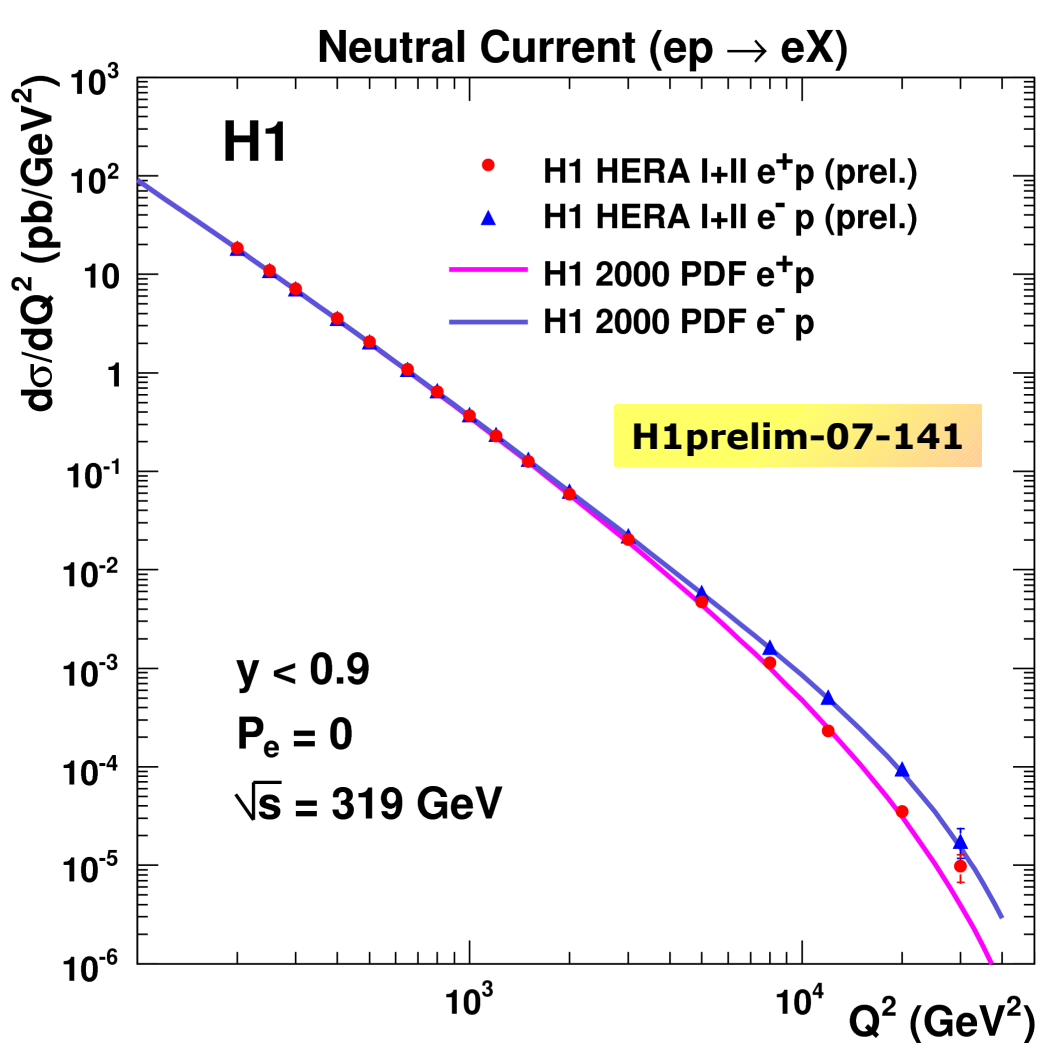

$$
x \tilde{F}_{3}=\frac{Y_{+}}{2 Y_{-}}\left(\tilde{\sigma}^{e^{-} p}-\tilde{\sigma}^{e^{+} p}\right) \approx \frac{x}{3}\left(2 u_{v}+d_{v}\right)
$$

$$
\sigma_{r}^{e^{ \pm} p \rightarrow e^{ \pm} X}=F_{2}-\frac{y^{2}}{Y_{+}} F_{L} \mp \frac{Y_{-}}{Y_{+}} x F_{3} \quad \text { ZEUS }
$$

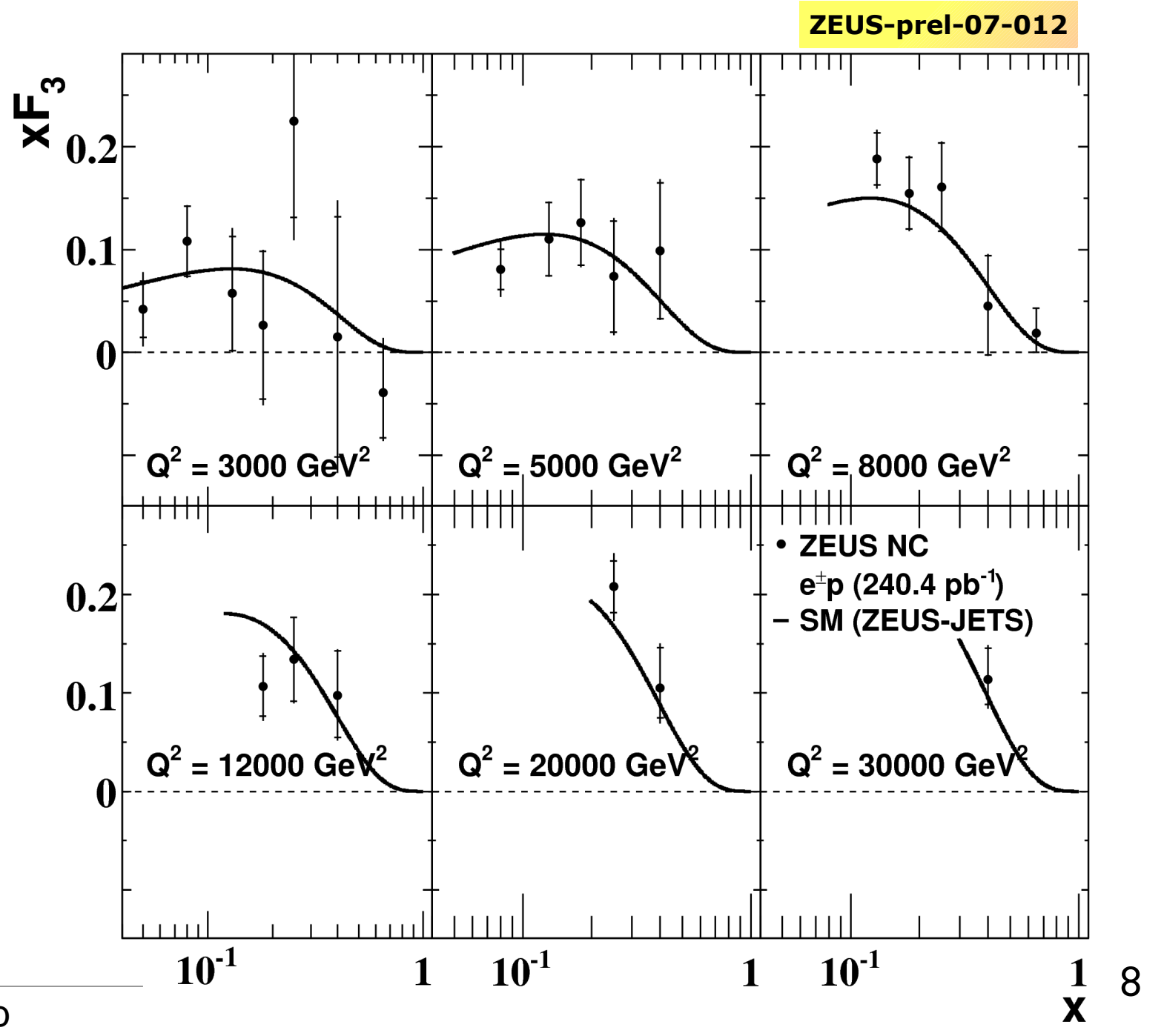

Proton Structure from HERA, Michał Własenko

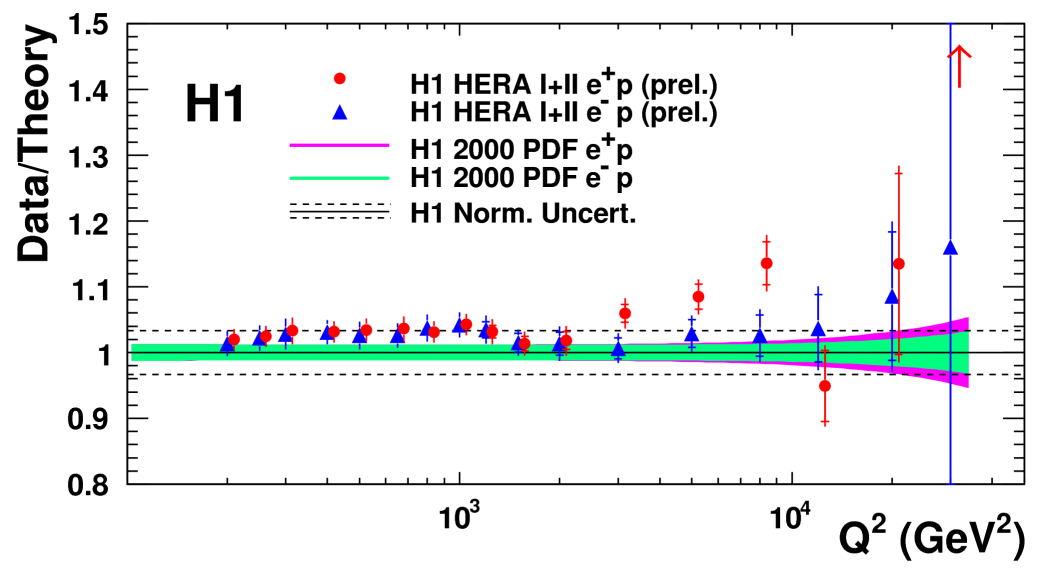




\section{High $Q^{2} N C$ with polarized $e^{ \pm}$}

$$
\begin{aligned}
& \tilde{F}_{2}=F_{2}^{\gamma}-\left(v_{e}-P_{e} a_{e}\right) \chi_{Z} F_{2}^{\gamma Z}+\left(v_{e}^{2}+a_{e}^{2}-2 P_{e} v_{e} a_{e}\right) \chi_{Z}^{2} F_{2}^{Z} \\
& x \tilde{F}_{3}=-\left(a_{e}-P_{e} v_{e}\right) \chi_{Z} x F_{3}^{\gamma Z}+\left(2 v_{e} a_{e}-P_{e}\left(v_{e}^{2}+a_{e}^{2}\right)\right) \chi_{Z}^{2} x F_{3}^{Z}
\end{aligned}
$$

- Polarization asymmetry provides direct measure for the EW effects

$$
\begin{aligned}
& A^{ \pm}=\frac{2}{P_{e}^{+}-P_{e}^{-}} \frac{\sigma^{ \pm}\left(P_{e}^{+}\right)-\sigma^{ \pm}\left(P_{e}^{-}\right)}{\sigma^{ \pm}\left(P_{e}^{+}\right)+\sigma^{ \pm}\left(P_{e}^{-}\right)} \\
& A^{ \pm} \simeq \mp \chi_{Z} a_{e} \frac{F_{2}^{\gamma Z}}{F_{2}^{\gamma}} \propto a_{e} v_{q}
\end{aligned}
$$

- $\boldsymbol{A}^{ \pm}$at large $\boldsymbol{x}$ also measures $\boldsymbol{d}_{\boldsymbol{v}} / \boldsymbol{u}_{\boldsymbol{v}}$ $A^{ \pm} \simeq \pm \chi_{Z} \frac{1+d_{v} / u_{v}}{4+d_{v} / u_{v}}$

Proton Structure from HERA, Michał Własenko

\section{ZEUS}

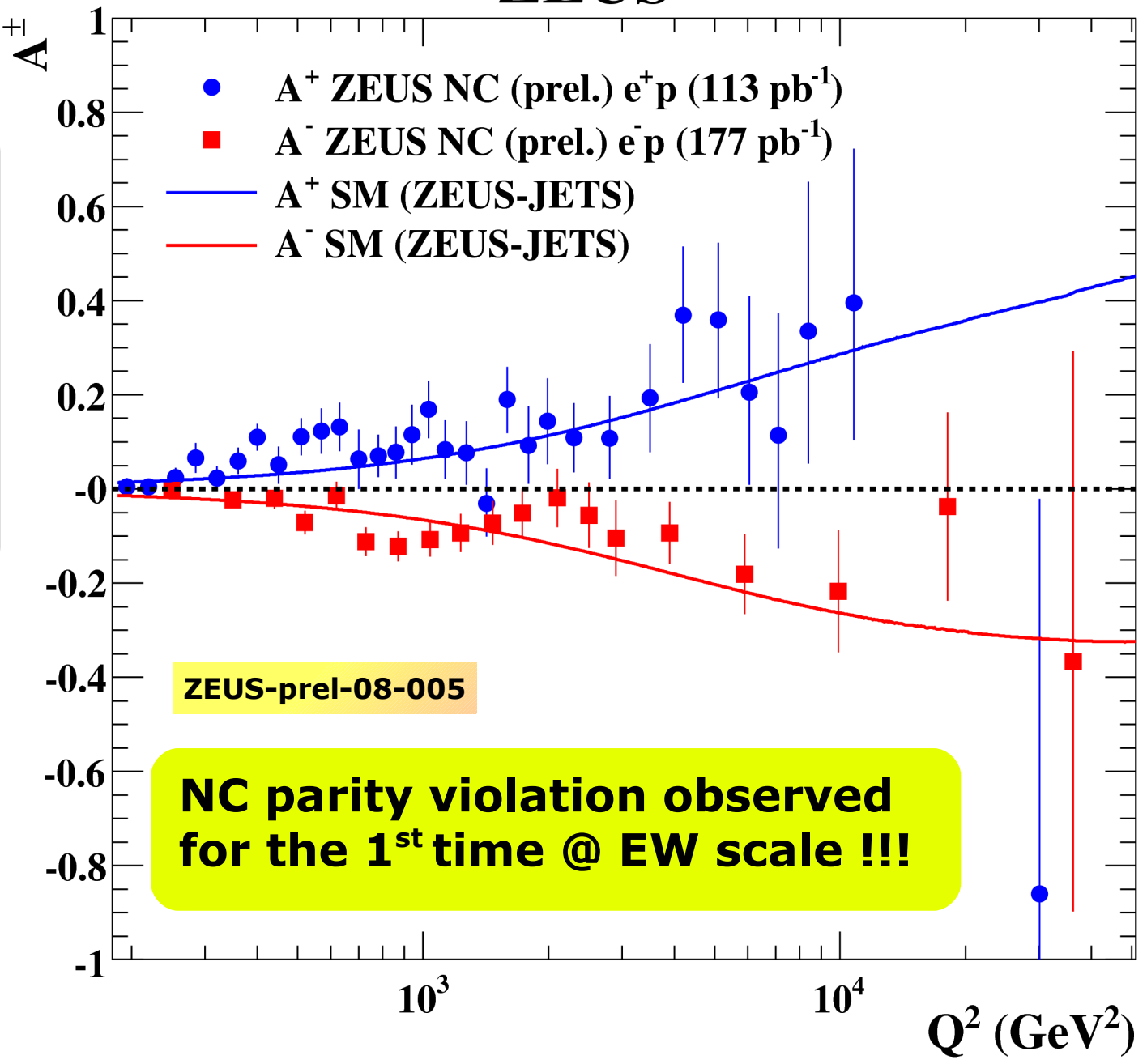




\section{CC cross sections}

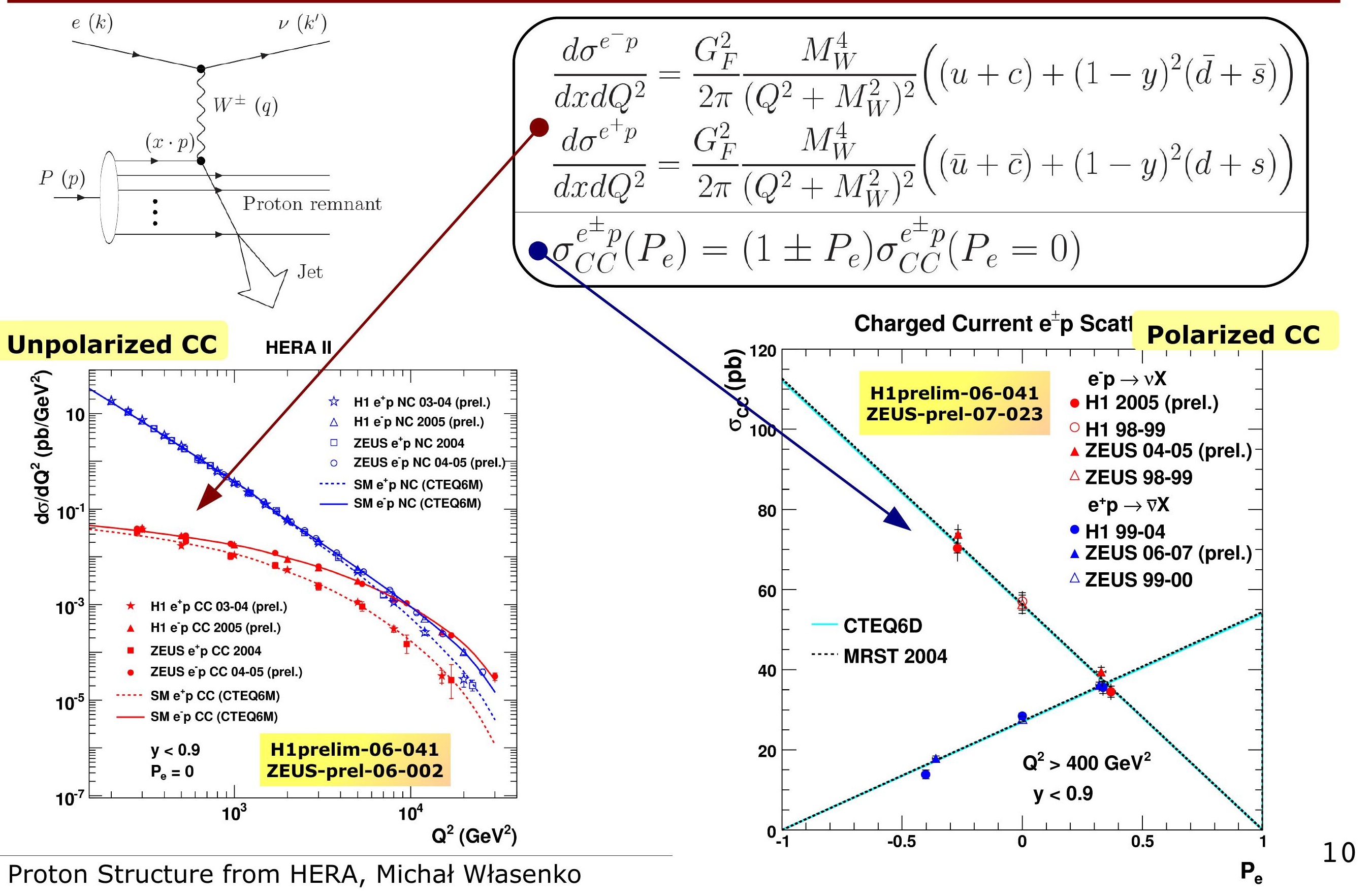




\section{Measurements of $F_{L}$}

$$
\begin{gathered}
\sigma_{r}\left(x, Q^{2}, y\right)=F_{2}\left(x, Q^{2}\right)-\frac{y^{2}}{Y_{+}} \cdot F_{L}\left(x, Q^{2}\right) \Rightarrow F_{L}\left(x, Q^{2}\right)=\frac{\partial \sigma_{r}\left(x, Q^{2}, y\right)}{\partial\left(y^{2} / Y_{+}\right)} \\
y=\frac{Q^{2}}{x s}
\end{gathered}
$$

- Indirect measurements of $\boldsymbol{F}_{\mathbf{L}}$ performed on previous HERA data by $\mathrm{H} 1$ (model-dependent)

- First ever direct $\boldsymbol{F}_{\mathbf{L}}$ measurements performed by varying $\sqrt{ } \mathbf{s}: 318,251,225 \mathrm{GeV}$

- Acceptances: ZEUS $\left(y<0.8, Q^{2}>24 \mathrm{GeV}^{2}\right), \mathrm{H} 1\left(y<0.9, Q^{2}>12 \mathrm{GeV}^{2}\right)$

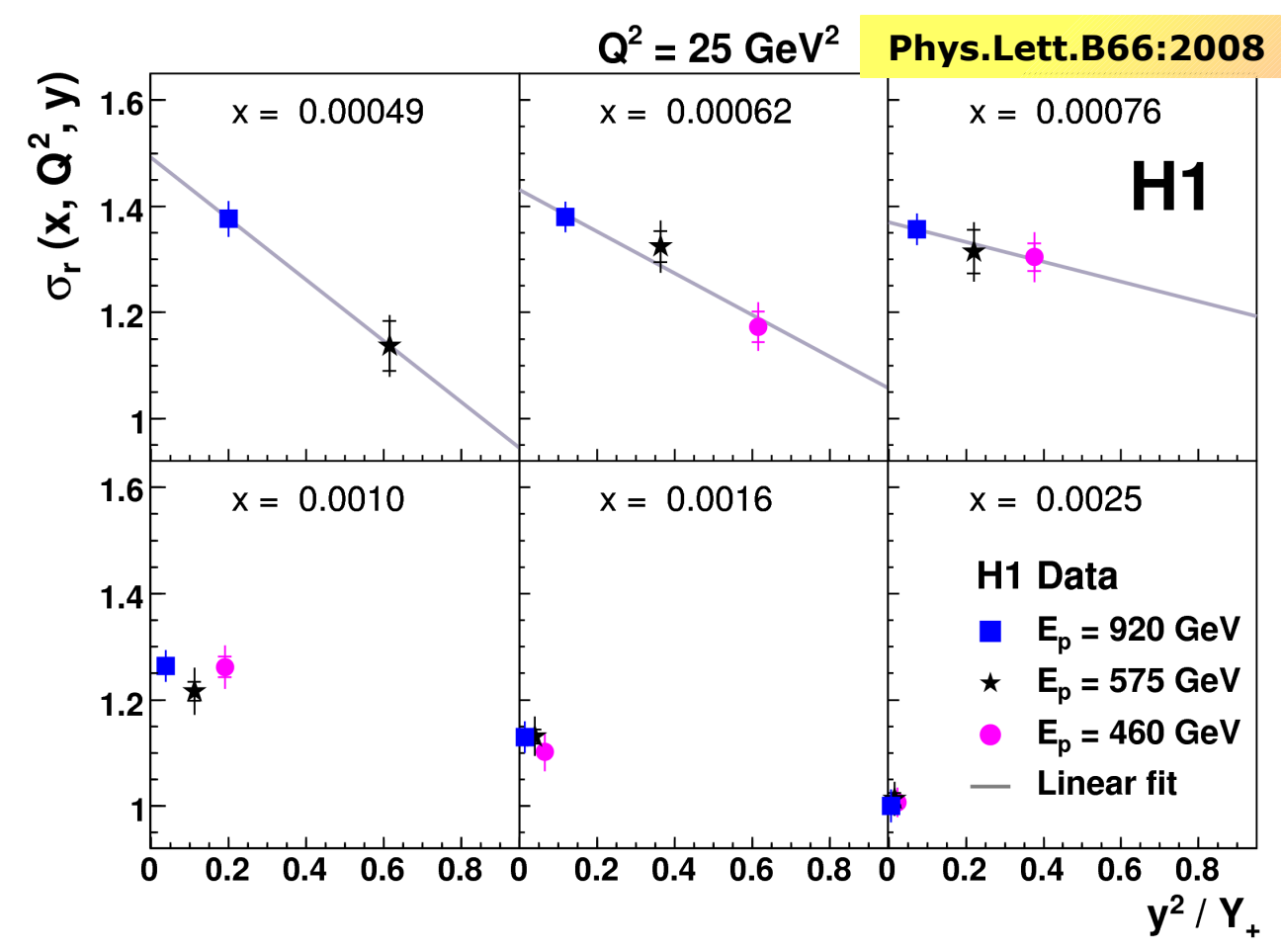

Proton Structure from HERA, Michał Własenko

\section{ZEUS}

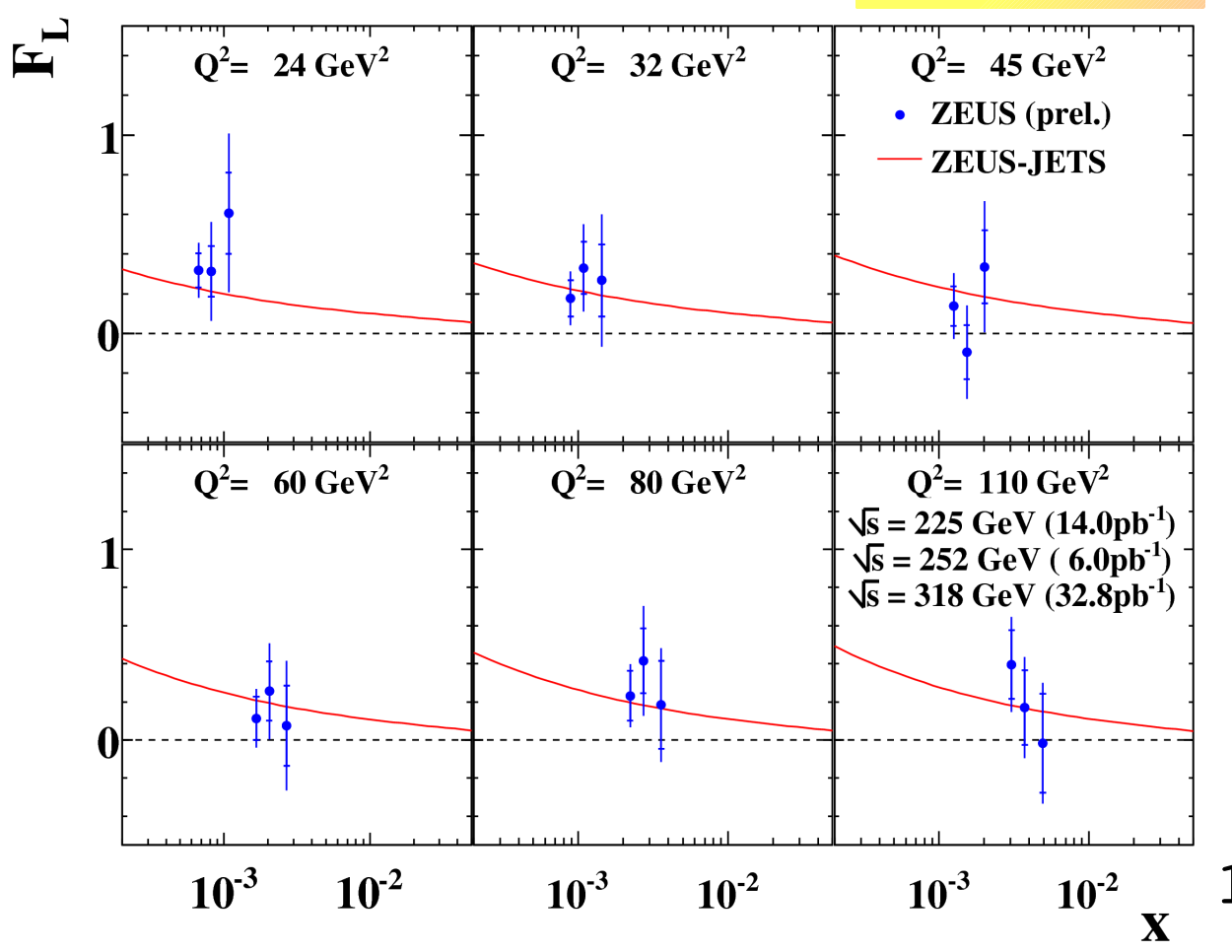




\section{Measurements of $F_{L}$}

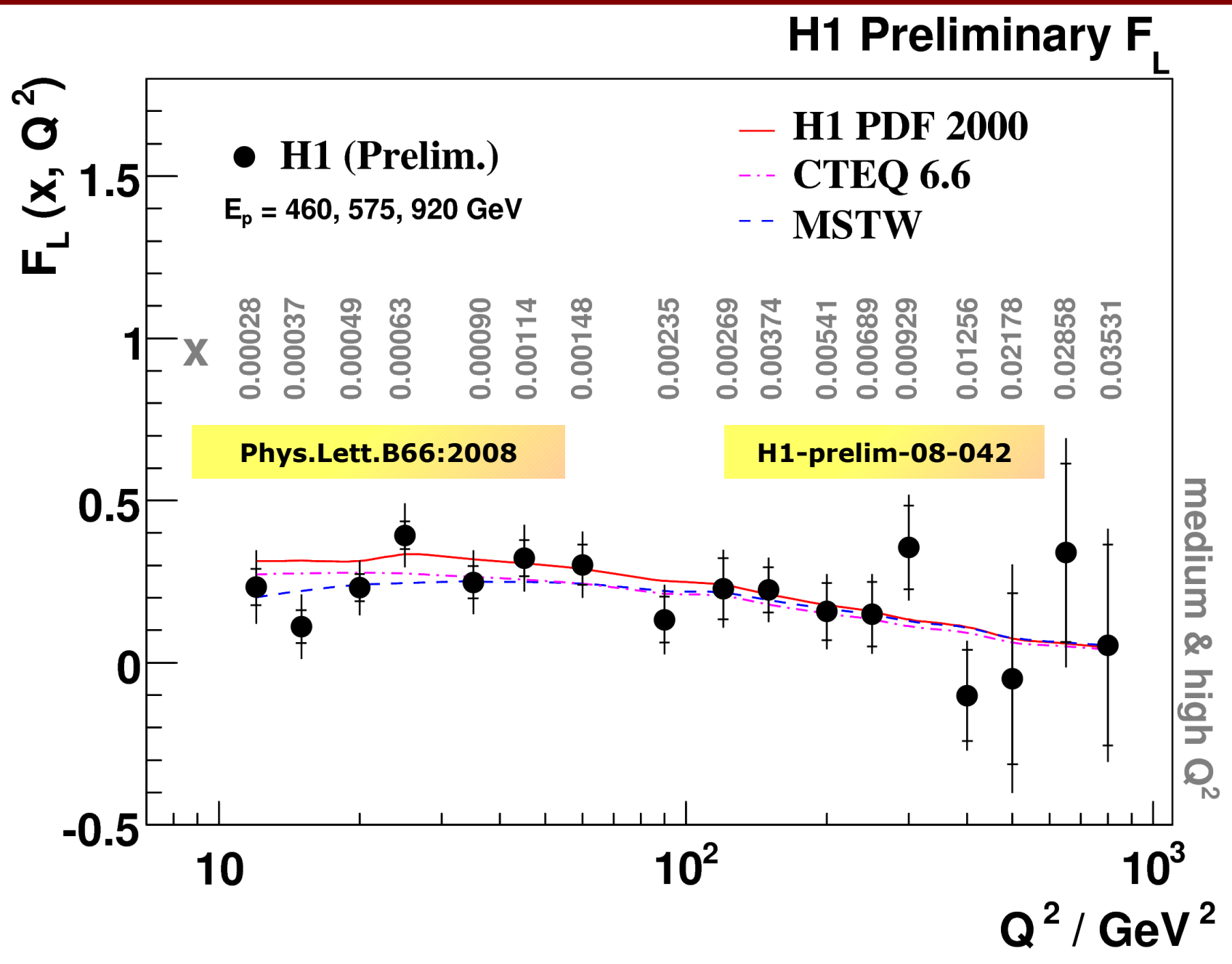

- Both results consistent with each other, and $\boldsymbol{F}_{\mathbf{L}} \neq \mathbf{0}$

- $\mathrm{H} 1$ 's averaged over $x$ results consistent with predictions from $\mathrm{pQCD}$

- $\boldsymbol{F}_{\boldsymbol{L}}$ confirms expectations on the behavior of $\boldsymbol{g}\left(\boldsymbol{x}, \boldsymbol{Q}^{2}\right)$ (coming from $\boldsymbol{F}_{\mathbf{2}}$ ) 


\section{Summary and outlook}

- $\mathrm{H} 1$ and ZEUS combined the inclusive NC results from HERAI data

- HERA combined PDF fits (available as HERAPDF library via LHAPDF) achieved unprecedented precision

- Middle and high $Q^{2}$ NC and CC HERAII data are still being analyzed and published

- First direct $\boldsymbol{F}_{\mathbf{L}}$ measurement published this Summer (can be expected to further constrain the low $x$ pQCD description)

- All inclusive results in excellent agreement with SM

- HERA approaching its 'final word' $\left(1 \mathrm{fb}^{-1}\right)$, producing priceless input for Tevatron and the LHC 
backup 


\section{The H1 \& ZEUS detectors}

(1ib)

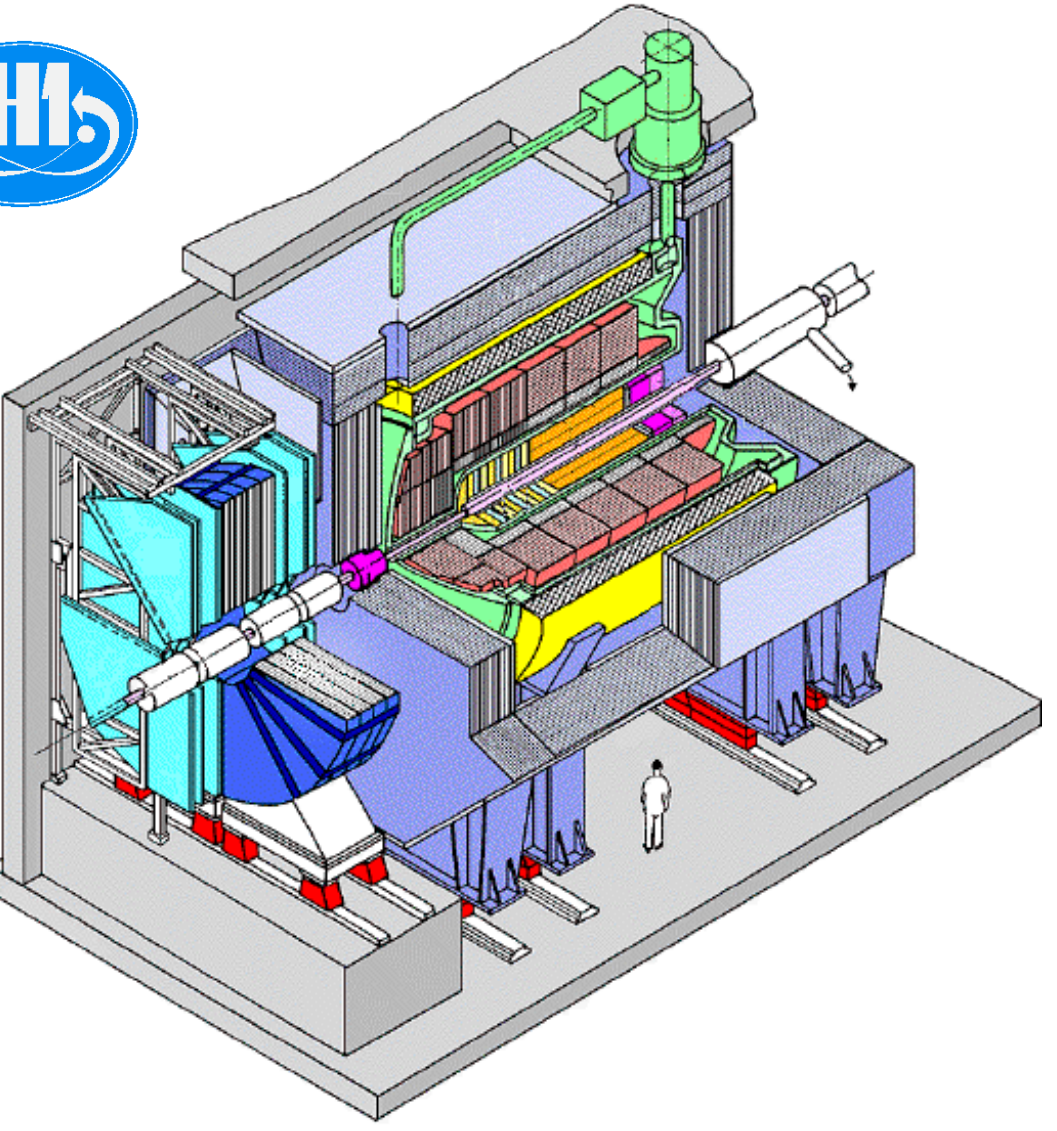

H1 calorimeters (45000 cells)

- $\sigma(E) / E=0.07 / \sqrt{ } E(E M$, small $\theta)$

- $\sigma(E) / E=0.12 / \sqrt{ } E(E M$, central $\theta)$

- $\sigma(E) / E=0.5 / \sqrt{ } E(H A D)$

Central + forward + backward trackers

Proton Structure from HERA, Michał Własenko

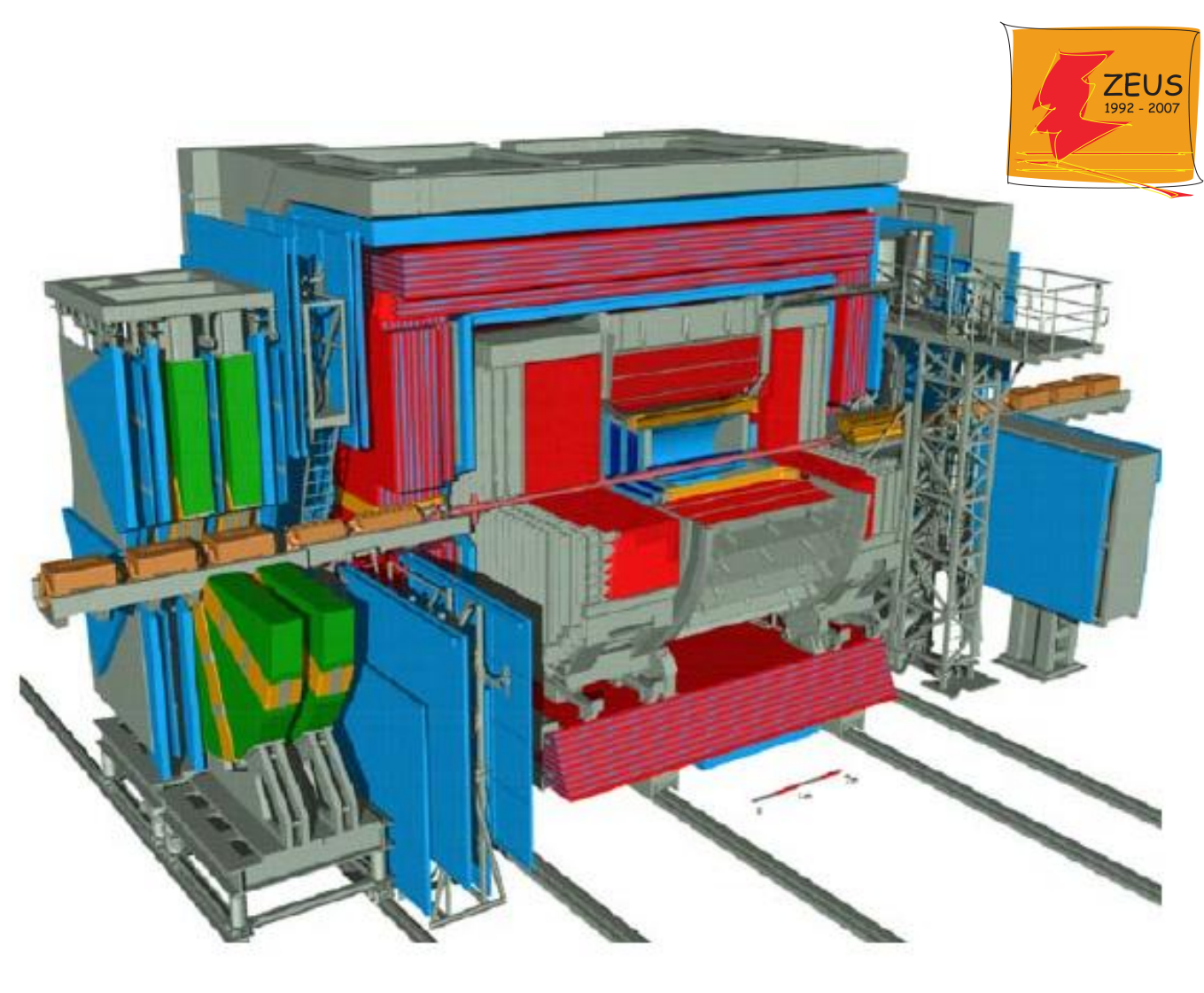

ZEUS calorimeter ( 12000 cells)

- $\sigma(E) / E=0.18 / \sqrt{ } E(E M)$

- $\sigma(E) / E=0.35 / \sqrt{ } E(H A D)$

Central + forward trackers 


\section{NC DIS event reconstruction}

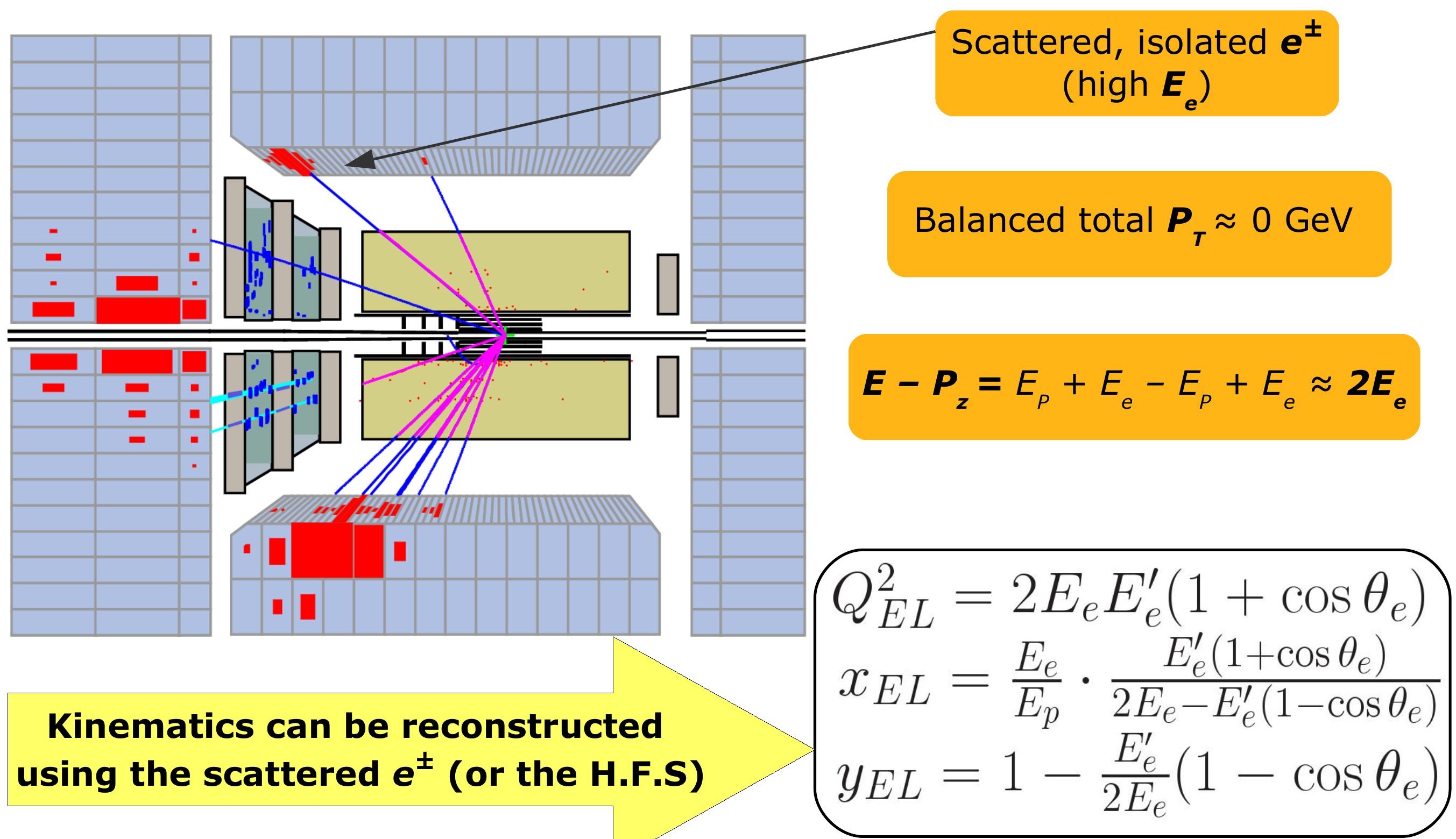

Proton Structure from HERA, Michał Własenko 


\section{(Example) predictions using HERAPDF0.1}
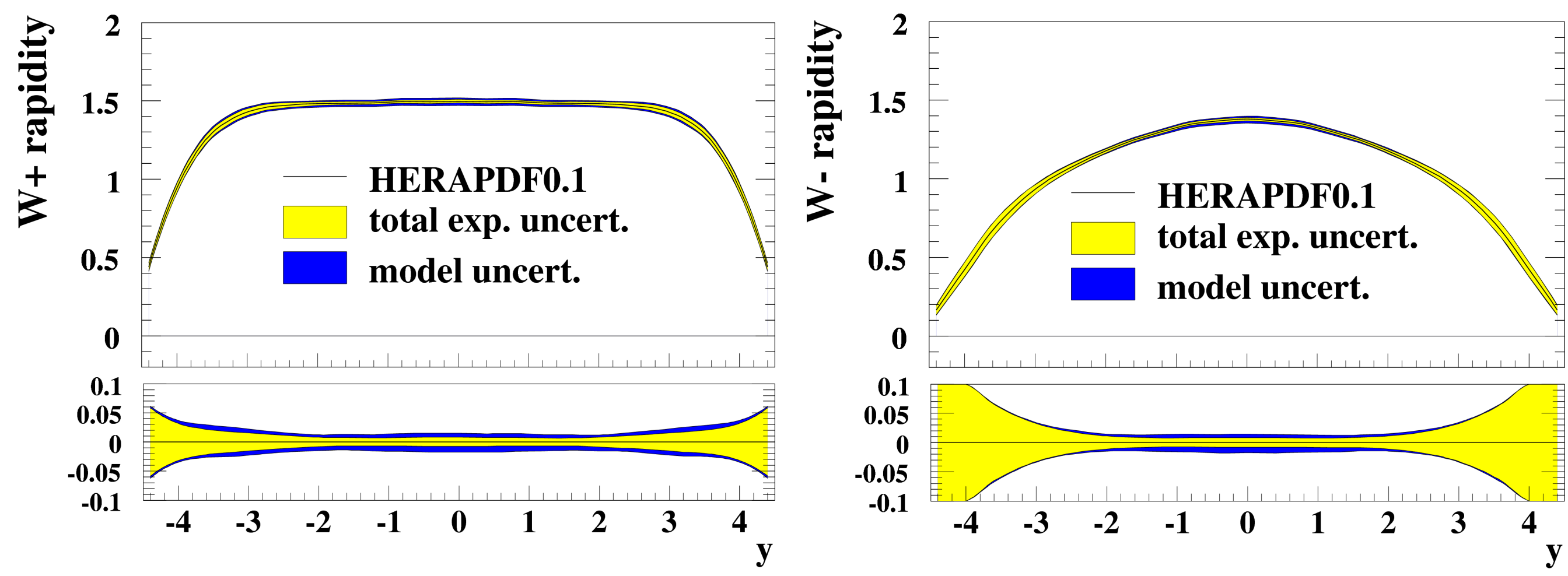

- Predicted rapidity distribution of $W+(W-)$ bosons in inclusive $W$ production at the LHC as predicted using only the HERA PDFs

- Small fractional error on the production 